Open Access

\title{
Network pharmacological identification of active compounds and potential actions of Erxian decoction in alleviating menopause-related symptoms
}

\author{
Shiwei Wang ${ }^{1}$, Yao Tong ${ }^{1}$, Tzi-Bun Ng${ }^{2}$, Lixing Lao', Jenny Ka Wing Lam³ , Kalin Yanbo Zhang ${ }^{1}$, Zhang-Jin Zhang ${ }^{1}$
} and Stephen Cho Wing Sze ${ }^{1^{*}}$

\begin{abstract}
Background: Erxian decoction (EXD) is used to treat menopause-related symptoms in Chinese medicine. This study aims to identify the bioactive compounds and potential actions of EXD by network pharmacological analysis.

Methods: Two databases, the Traditional Chinese Medicine Systems Pharmacology database and TCM Database@ Taiwan, were used to retrieve literature of phytochemicals of EXD. STITCH 4.0 and the Comparative Toxicogenomics Database were used to search for compound-protein and compound-gene interactions, respectively. DAVID Bioinformatics Resources 6.7 and Cytoscape 3.01 with Jepetto plugin software were used to perform a network pharmacological analysis of EXD.

Results: A total of 721 compounds were identified in EXD, of which 155 exhibited 2,656 compound-protein interactions with 1,963 associated proteins determined by STITCH4.0 database, and of which 210 had 14,893 compoundgene interactions with 8,536 associated genes determined by Comparative Toxicogenomics Database. Sixty three compounds of EXD followed the Lipinski's Rule with $O B \geq 30 \%$ and DL index $\geq 0.18$, of which 20 related to 34 significant pathway- or 12 gene- associated with menopause.
\end{abstract}

Conclusions: Twenty compounds were identified by network pharmacology as potential effective ingredients of EXD for relieving menopause with acceptable oral bioavailability and druggability.

\section{Background}

By the age of 35 years, the quality and quantity of ovarian follicles would decline [1], and consequential hormonal and symptomatic changes would lead to cessation of menses [2]. During menopause, the fluctuating levels of sex hormones, including luteinizing hormone, folliclestimulating hormone, estrogen, and progesterone [3], can cause osteoporosis and menopausal symptoms, such as hot flushes, depression, nocturnal sweating, uterine bleeding, vaginal dryness, insomnia, and loss of sexual function [4-6]. It is estimated that there will be about 1.2

\footnotetext{
*Correspondence: stephens@hku.hk

${ }^{1}$ School of Chinese Medicine, Li Ka Shing Faculty of Medicine, The

University of Hong Kong, Hong Kong SAR, China

Full list of author information is available at the end of the article
}

billion menopausal women worldwide by 2030 [7]. Menopause occurs between 44.6 and 52 years of age, varying among different races and countries [8]. In the United States, about 6,000 women reach menopause every day, which is more than 2 million per year [7]. The average age of menopause in the United Kingdom and United States is 52 and 51 years, respectively $[9,10]$. In China, women around 50 years of age would experience natural menopause and in the southeast of China reach menopause at an average age of 48.9 years [11, 12]; thus, 0.28 billion women will be over the age of 50 years by 2030 would have menopause [13].

Hormone replacement therapy (HRT) has been used for more than 60 years to relieve menopausal symptoms. However, there are many adverse effects associated with 
HRT [14], e.g., increasing the risks of breast cancer, coronary artery disease, endometrial cancer, venous thromboembolism and stroke [15].

Chinese medicines (CM) are also used in treating menopausal symptoms [16-21]. Some Chinese herbal formulas (CHFs) are indicative for treating gynecological disorders including menopausal symptoms [16, 17]. However, few studies on the biological actions of the CHFs have been conducted [24-26]. As a typical example for CHFs, Erxian decoction (EXD) is commonly used to treat menopause related symptoms [17, 22-34], consisting of six herbs, Herba Epimedium Brevicornum (HE; Xian-ling-pi), Rhizoma Curculiginis Orchioides (RC; Xian-mao), Radix Morindae Officinalis (RMO; Ba-jitian), Radix Angelicae Sinensis (RAS; Dang-gui), Cortex Phellodendri Chinensis (CPC; Huang-bo), and Rhizoma Anemarrhenae Asphodeloides (RA; Zhi-mu) [35].

During the past two decades, drug discovery has pursued a dominant target, "one drug, one disease" paradigm. However, many drugs exert therapeutic effects via restoration of multiple disease-related targets rather than a single one [36, 37]. Network pharmacology, which is based on systems biology, polypharmacology and molecular network analysis, provides a possible strategy to elucidate the action mechanism of multi-ingredient medicine in a holistic view [38-40]. Molecular networks are constructed by interactions of target-based proteins and genes for predicting their function and facilitating drug discovery, which provides pharmacological information in a holistic manner [40, 41]. Enrichment analysis is an analytical method to assess functional associations between sets of genes or proteins of interest to us and a database of known gene or protein sets $[42,43]$. It can identify the significant pathways and their enriched gene/protein sets, and elucidate significant multiple pharmacological mechanisms $[42,44]$.

The complexity of numerous chemical constituents and biological actions has not been fully identified in EXD. This study aims to identify the bioactive compounds and actions of EXD by a network pharmacological analysis.

\section{Methods}

The constituent compounds of EXD were identified by two phytochemical databases, the Traditional Chinese Medicine Systems Pharmacology (TCMSP) database and TCM Database@Taiwan., as well as published EXD literatures [26-30, 35, 45, 46]. The druggability analysis of the identified compounds in EXD were performed and provided by Lipinski's rule (LR) and TCMSP database in term of oral bioavailability (OB) and drug-likeness (DL) indices, respectively. $\mathrm{OB}$ is the degree to which a drug or other substance becomes available to the target tissue after oral administration. DL is to evaluate their potentials to be bioactive compounds compare with the well-developed drug. The significant pathways and gene-associated diseases for the identified compounds were determined by enrichment analysis (JEPETTO (US): http://apps.cytoscape.org/apps/jepetto) [43] of the compound-protein interaction and enrichment analysis (DAVID 6.7 (US): http://david.abcc.ncifcrf.gov/home.jsp) [47] of the compound-gene interactions, respectively. The workflow of the network pharmacology study of EXD was summarized in Figure 1.

\section{Identification of potential bioactive constituents in EXD}

All phytochemicals from the six constituent herbs of EXD were identified by the TCM Database@Taiwan (http://tcm.cmu.edu.tw/), TCMSP database (http:// sm.nwsuaf.edu.cn/lsp/tcmsp.php), and previous EXD literatures [26-30, 35, 45, 46].

\section{Druggability analysis by LR, OB and DL properties}

Lipinski's rule (LR) [48] was used to identify druggable compounds according to the following criteria: molecular weight $(\mathrm{MW})$ of not more than $500 \mathrm{Da}(\mathrm{MW} \leq 500)$, chemical composition with no more than five hydrogen bond donors ( $\mathrm{H}$-bond donors $\leq 5$ ), no more than 10 hydrogen bond acceptors ( $\mathrm{H}$-bond acceptors $\leq 10$ ), and octanol-water partition coefficient, LogP, no $>5$ $(\log \mathrm{P} \leq 5)$. A compound that does not satisfy at least two of the above conditions is less likely to be an orally active drug [49].

The phytochemical information of the compounds with their $\mathrm{OB}$ and DL properties were explored using the TCMSP database, which embed OBioavail 1.1 software for OB [50] and Tanimoto similarity software for DL [51]. The DL calculations in TCMSP database were based on the following formula [51]:

$$
F(A, B)=A \times \frac{B}{A^{2}+B^{2}-A \times B}
$$

where $A$ is related to the molecular property of the target compound and $B$ refers to the average molecular properties of all drugs from the Drugbank database (http://www. drugbank.ca/). A more detailed calculation of the DL index can be found in Tao et al. [51] and Wang et al. [52]. The thresholds used were $\mathrm{OB} \geq 30 \%$ and DL index $\geq 0.18$, as recommended by the TCMSP database. The thresholds were selected to efficiently identify bioactive compounds from the large pool of chemical compounds based on the following criteria: (1) the model obtained could be reasonably explained by previous pharmacological data and (2) the compound met the recommended mean DL index of 0.18 (the mean of DL index of 6,511 molecules from Drugbank database (2011) is 0.18) [51, 52]. 


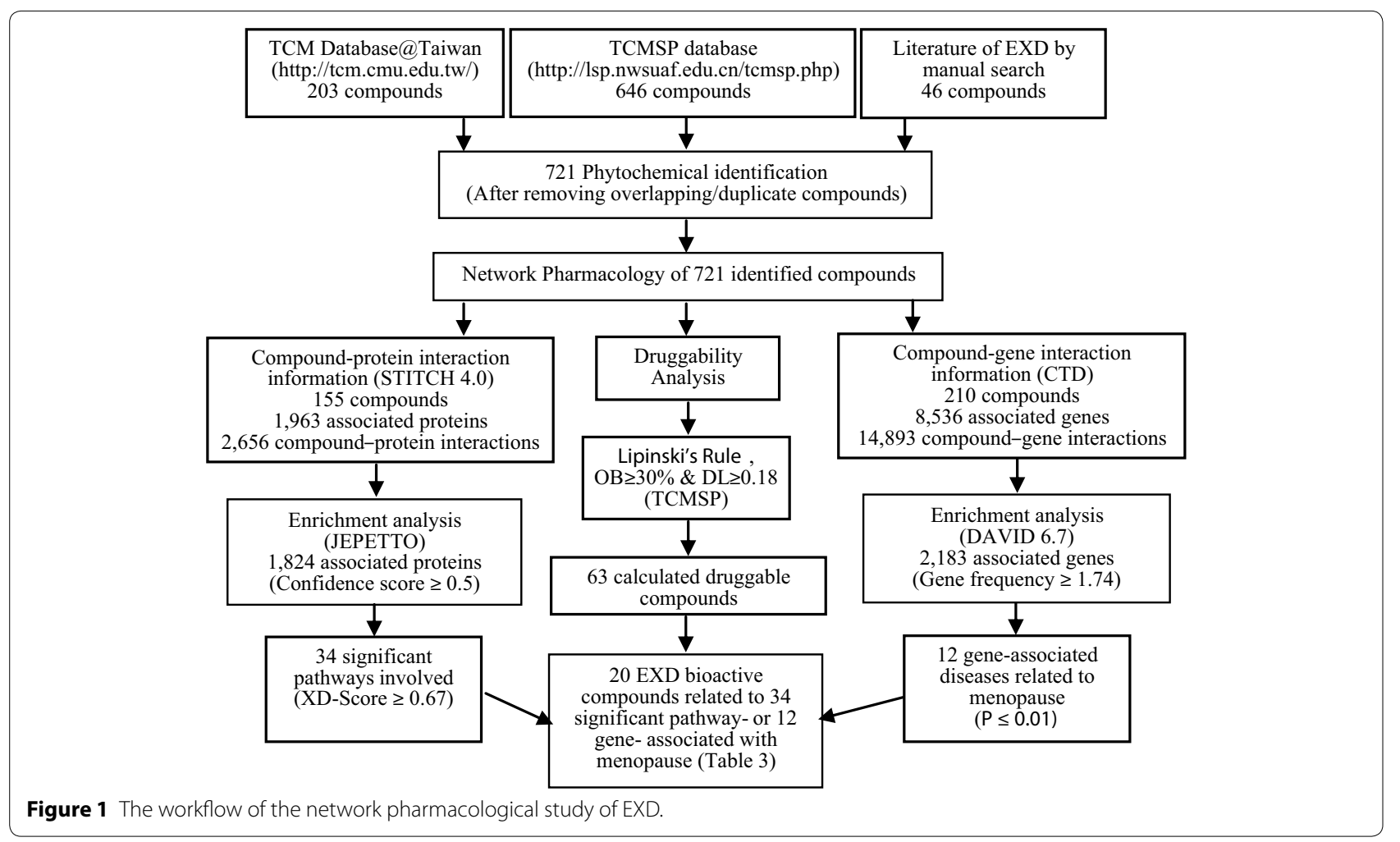

\section{Identification of associated proteins and genes}

The integrative efficacy of the identified constituents in EXD was determined by analyzing the chemical-protein and chemical-gene interactions obtained from the Search Tool for Interactions of Chemicals and Proteins (STITCH) database and Comparative Toxicogenomics Database (CTD), respectively. The STITCH 4.0 database (http://stitch.embl.de/) can be used to study potential interactions between 300,000 phytochemicals and 2.6 million proteins curated from 1,133 organisms [53]. In this database, the approximate probability of a predicted association for a chemical-protein interaction is determined by the confidence score, with a higher score indicating a stronger interaction (low confidence score $\sim 0.2$; medium confidence score $\sim 0.5$; high confidence score $\sim 0.75$; highest confidence score $\sim 0.95$, provided by STITCH 4.0 database). The CTD (http://ctd.mdibl.org/) is a publicly available research resource that includes more than 116,000 interactions between 9,300 chemicals and 13,300 genes [54]. Both databases were searched independently by two researchers to minimize any bias.

In order to identify the associated significant pathways, proteins with a chemical-protein interaction confidence score $\geq 0.5$ were selected for the enrichment analysis by JEPETTO with the KEGG database, a Java-based Cytoscape 3.01 plugin [43]. For studying the gene-associated diseases, the genes were firstly ranked by frequency of occurrence of the chemical-gene interactions, and then the genes with gene frequency $\geq 1.67$ were chosen for the enrichment analysis by Visualization and Integrated Discovery (DAVID) Bioinformatics Resources 6.7 (http://david.abcc.ncifcrf.gov/).

\section{Results}

\section{Compounds in EXD}

Eight hundred and ninety-five phytochemicals were collected from the six herbs in EXD. From the TCM Database@Taiwan, 203 compounds were identified, comprising 29 in HE, 44 in RC, 38 in RMO, 56 in RAS, seven in CPC, and 29 in RA. From the TCMSP database, 646 compounds were identified, comprising 130 in $\mathrm{HE}, 78$ in $\mathrm{RC}, 174$ in RMO, 125 in RAS, 58 in CPC, and 81 in RA. 46 phytochemicals from previous studies in the literature [26-30, 35, 45, 46], comprising 15 in $\mathrm{HE}$, one in $\mathrm{RC}$, five in ROM, five in RAS, 14 in CPC, 5 in RA, and one in EXD (specific herbs unknown). Finally, a total of 721 phytochemicals were identified in EXD after removing overlapping/duplicate compounds from the databases and the literature (Additional file 1).

\section{Identifying druggable compounds by LR, OB, and DL predictions}

Of the 150 compounds from HE, 75 (50\%) compounds were identified based on LR, 23 (15.3\%) had OB $\geq 30 \%$ 
and DL index $\geq 0.18$, and only 17 (11.3\%) satisfied all criteria. Of the 104 compounds from RC, 29 (27.9\%) passed LR, seven (6.7\%) had $\mathrm{OB} \geq 30 \%$ and DL index $\geq 0.18$, and only four (3.8\%) satisfied all criteria. Of the 189 compounds from RMO, 125 (66.1\%) passed LR, 20 (10.6\%) had OB $\geq 30 \%$ and DL index $\geq 0.18$, and only $12(6.3 \%)$ satisfied all criteria. Of the 173 compounds from RAS, 131 (75.7\%) passed LR, five (2.9\%) had OB $\geq 30 \%$ and DL index $\geq 0.18$, and only three (1.7\%) satisfied all criteria. Of the 63 compounds from CPC, 43 (68.3\%) passed LR, 28
(44.4\%) had OB $\geq 30 \%$ and DL index $\geq 0.18$, and only 19 (30.2\%) satisfied all criteria. Of the 81 compounds from RA, 45 (55.6\%) passed LR, 15 (18.5\%) had OB $\geq 30 \%$ and DL index $\geq 0.18$, and only $11(13.6 \%)$ satisfied all criteria (Table 1). The physicochemical properties of anemarsaponin BII from EXD reported in the literature (specific herbs unknown) did not pass LR. Overall, 66 compounds passed LR and had $\mathrm{OB} \geq 30 \%$ and DL index $\geq 0.18$. A total of 63 compounds were obtained after removing the duplicate compounds (Table 2).

Table 1 Compounds in EXD satisfying $L R, O B \geq 30 \%$ and $D L \geq 0.18$

\begin{tabular}{lllllll}
\hline & Herbs & & & \\
\cline { 2 - 7 } & HE & RC & RMO & RAS & CPC & RA \\
\hline Number of compounds & 150 & 104 & 189 & 173 & 63 & 81 \\
Compounds (percentage) passing $L R$ & $75(50.0 \%)$ & $29(27.9 \%)$ & $125(66.1 \%)$ & $131(75.7 \%)$ & $43(68.3 \%)$ & $45(55.6 \%)$ \\
Compounds (percentage) with $\mathrm{OB} \geq 30 \%$ and $\mathrm{DL} \geq 0.18$ & $23(15.3 \%)$ & $7(6.7 \%)$ & $20(10.6 \%)$ & $5(2.9 \%)$ & $28(44.4 \%)$ & $15(18.5 \%)$ \\
Compounds (percentage) satisfying $\mathrm{LR}, \mathrm{OB} \geq 30 \%$ and $\mathrm{DL} \geq 0.18$ & $17(11.3 \%)$ & $4(3.8 \%)$ & $12(6.3 \%)$ & $3(1.7 \%)$ & $19(30.2 \%)$ & $11(13.6 \%)$ \\
\hline
\end{tabular}

$L R$ Lipinski's rule, $O B$ oral bioavailability, DL drug-likeness index, HE Herba Epimedium Brevicornum, RC Rhizoma Curculiginis Orchioides, RMO Radix Morindae Officinalis, RAS Radix Angelicae Sinensis, CPC Cortex Phellodendri Chinensis, RA Rhizoma Anemarrhenae asphodeloides.

Table 2 The 63 bioactive compounds from HE, RC, RMO, RAS, CPC, and RA herbs and their corresponding molecular properties, OB and DL (20 of 63 bioactive compounds related to 34 significant pathway- or 12 gene- associated with menopause)

\begin{tabular}{|c|c|c|c|c|c|c|c|}
\hline Phytochemical & MW & AlogP & Hdon & Hacc & OB (\%) & DL & Herb \\
\hline 1. DFV((2S)-7-hydroxy-2-(4-hydroxyphenyl)chroman-4-one) ${ }^{b}$ & 256.27 & 2.57 & 2 & 4 & 32.76 & 0.18 & $\mathrm{HE}$ \\
\hline 2. Delta7-dehydrosophoramine & 242.35 & 1.09 & 0 & 3 & 54.45 & 0.25 & CPC \\
\hline 3. Alizarin-2-methylether & 254.25 & 2.53 & 1 & 4 & 32.81 & 0.21 & $\mathrm{RMO}$ \\
\hline 4. 1-Hydroxy-3-methoxy-9,10-anthraquinone & 254.25 & 2.53 & 1 & 4 & 104.33 & 0.21 & $\mathrm{RMO}$ \\
\hline 5. 1-Hydroxy-6-hydroxymethylanthracenequinone & 254.25 & 1.94 & 2 & 4 & 81.77 & 0.21 & $\mathrm{RMO}$ \\
\hline 6. Skimmianin (4,7,8-trimethoxyfuro[2,3-b]quinoline) & 259.28 & 2.33 & 0 & 5 & 40.14 & 0.20 & CPC \\
\hline $\begin{array}{l}\text { 7. Magnograndiolide ((3aS,6R,6aR,9R,9aS,9bS)-6,9-dihydroxy-6,9-dimethyl-3-methyl- } \\
\text { idene-3a,4,5,6a,7,8,9a,9b-octahydroazuleno[5,4-d]furan-2-one) }\end{array}$ & 266.37 & 1.18 & 2 & 4 & 63.71 & 0.19 & $\mathrm{HE} / \mathrm{CPC}$ \\
\hline 8. Coumaroyltyramine (cis- $N$-p-Coumaroyltyramine) & 283.35 & 2.88 & 3 & 4 & 112.9 & 0.20 & RA \\
\hline 9. Kaempferol(3,5,7-trihydroxy-2-(4-hydroxyphenyl)chromen-4-one) ${ }^{\text {b }}$ & 286.25 & 1.77 & 4 & 6 & 41.88 & 0.24 & $\mathrm{HE} / \mathrm{RA}$ \\
\hline 10. Luteolin(2-(3,4-dihydroxyphenyl)-5,7-dihydroxychromen-4-one) ${ }^{b}$ & 286.25 & 2.07 & 4 & 6 & 36.16 & 0.25 & $\mathrm{HE}$ \\
\hline 11. Rutaecarpine(Indolo $\left(2^{\prime}, 3^{\prime}: 3,4\right)$ pyrido(2,1-b)quinazolin-5(7H)-one, 8,13-dihydro-(9Cl) $)^{b}$ & 287.34 & 3.36 & 1 & 3 & 40.30 & 0.60 & CPC \\
\hline 12. 8-(3-methylbut-2-enyl)-2-phenyl-chromone & 290.38 & 4.99 & 0 & 2 & 48.54 & 0.25 & $\mathrm{HE}$ \\
\hline 13. Dehydrotanshinone II A (1,6,6-trimethyl-7H-naphtho[5,6-g] [1] benzoxole-10,11-dione) & 292.35 & 4.22 & 0 & 3 & 43.76 & 0.40 & $\mathrm{CPC}$ \\
\hline 14. Chryseriol(5,7-dihydroxy-2-(4-hydroxy-3-methoxyphenyl)chromen-4-one) ${ }^{b}$ & 300.28 & 2.32 & 3 & 6 & 35.85 & 0.27 & HE \\
\hline 15. Phellopterin(4-methoxy-9-(3-methylbut-2-enoxy)furo[3,2-g]chromen-7-one) ${ }^{b}$ & 300.33 & 3.64 & 0 & 5 & 40.19 & 0.28 & $\mathrm{CPC}$ \\
\hline 16. Cnidilin(9-methoxy-4-(3-methylbut-2-enoxy)furo[3,2-g]chromen-7-one) ${ }^{\text {b }}$ & 300.33 & 3.64 & 0 & 5 & 32.69 & 0.28 & RAS \\
\hline 17. Quercetin(2-(3,4-dihydroxyphenyl)-3,5,7-trihydroxychromen-4-one) ${ }^{b}$ & 302.25 & 1.50 & 5 & 7 & 46.43 & 0.28 & $\mathrm{HE}$ \\
\hline 18. (Z)-3-(4-hydroxy-3-methoxy-phenyl)-N-[2-(4-hydroxyphenyl)ethyl]acrylamide & 313.38 & 2.86 & 3 & 5 & 118.35 & 0.26 & RA \\
\hline 19. 1,6-dihydroxy-5-methoxy-2-(methoxymethyl)-9,10-anthraquinone & 314.31 & 2.06 & 2 & 6 & 104.54 & 0.34 & $\mathrm{RMO}$ \\
\hline 20. Hippeastrine(Lycorenan-7-one, 5-hydroxy-1-methyl-9,10-(methylenebis(oxy))-, (5alpha)-) & 315.35 & 1.17 & 1 & 6 & 51.65 & 0.62 & RA \\
\hline 21. Coptisine(6,7-dihydro-bis(1,3)benzodioxolo (5,6-a:4',5'-g)quinolizinium) & 320.34 & 3.25 & 0 & 4 & 30.67 & 0.86 & $\mathrm{CPC}$ \\
\hline 22. 1,2-bis(4-hydroxy-3-methoxyphenyl)propan-1,3-diol & 320.37 & 1.69 & 4 & 6 & 52.31 & 0.22 & $\mathrm{HE}$ \\
\hline 23. 2-Hydroxy-1,5-dimethoxy-6-(methoxymethyl)-9,10-anthraquinone & 328.34 & 2.31 & 1 & 6 & 95.85 & 0.37 & $\mathrm{RMO}$ \\
\hline 24. 2-Hydroxy-1,8-dimethoxy-7-methoxymethylanthracenequinone & 328.34 & 2.31 & 1 & 6 & 112.30 & 0.37 & $\mathrm{RMO}$ \\
\hline
\end{tabular}


Table 2 continued

\begin{tabular}{|c|c|c|c|c|c|c|c|}
\hline Phytochemical & MW & AlogP & Hdon & Hacc & $\mathrm{OB}(\%)$ & $\mathrm{DL}$ & Herb \\
\hline 25. Americanin $A^{a}$ & 328.34 & 2.30 & 3 & 6 & 46.71 & 0.35 & $\mathrm{RMO}$ \\
\hline 26. C-Homoerythrinan, 1,6-didehydro-3,15,16-trimethoxy-, (3.beta.) & 329.48 & 2.89 & 0 & 4 & 39.14 & 0.49 & HE \\
\hline 27. 1,5,7-Trihydroxy-6-methoxy-2-methoxymethylanthracenequinone & 330.31 & 1.79 & 3 & 7 & 80.42 & 0.38 & $\mathrm{RMO}$ \\
\hline 28. (2R,3S)-(+)-3',5-Dihydroxy-4,7-dimethoxydihydroflavonol & 332.33 & 1.99 & 3 & 7 & 77.24 & 0.33 & $\mathrm{RMO}$ \\
\hline 29. 2-Hydroxyethyl 5-hydroxy-2-(2-hydroxybenzoyl)-4-(hydroxymethyl)benzoate & 332.33 & 1.41 & 4 & 7 & 62.32 & 0.26 & $\mathrm{RMO}$ \\
\hline 30. Chelerythrine $a, b$ & 332.37 & 4.29 & 0 & 4 & 34.18 & 0.78 & CPC \\
\hline 31. Worenine ${ }^{a}$ & 334.37 & 3.73 & 0 & 4 & 45.83 & 0.87 & CPC \\
\hline 32. Yinyanghuo $C(2-(2,2$-dimethylchromen-6-yl)-5,7-dihydroxychromen-4-one) & 336.36 & 3.39 & 2 & 5 & 45.67 & 0.50 & HE \\
\hline 33. Berberine $e^{a, b}$ & 336.39 & 3.45 & 0 & 4 & 36.86 & 0.78 & CPC \\
\hline $\begin{array}{l}\text { 34. Isocorypalmine ((13aS)-5,8,13,13a-tetrahydro-3,9,10-trimethoxy-6H-Dibenzo[a,g]quinolizin- } \\
\text { 2-ol) }\end{array}$ & 341.44 & 3.35 & 1 & 5 & 35.77 & 0.59 & CPC \\
\hline 35. Yinyanghuo E (5,7-dihydroxy-2-(8-hydroxy-2,2-dimethylchromen-6-yl)chromen-4-one) & 352.36 & 3.12 & 3 & 6 & 51.63 & 0.55 & $\mathrm{HE}$ \\
\hline 36. Palmatine(Palmatine chloride is another name in TCMSP database) ${ }^{\text {b }}$ & 352.44 & 3.65 & 0 & 4 & 64.6 & 0.65 & CPC \\
\hline $\begin{array}{l}\text { 37. Fumarine(7-methyl-6,8,9,16-tetrahydrobis[1, 3]benzodioxolo[4,5-c:5',6'-g]azecin-15(7H)- } \\
\text { one) })^{\mathrm{b}}\end{array}$ & 353.40 & 2.95 & 0 & 6 & 59.26 & 0.83 & CPC \\
\hline $\begin{array}{l}\text { 38. Cavidine(9-dimethoxy-6-methyl-6,6a,11,14-tetrahydro-8,12H- } \\
\text { benzo(a)-1,3-benzodioxolo(4,5-g)quinolizine) }\end{array}$ & 353.45 & 3.72 & 0 & 5 & 35.64 & 0.81 & CPC \\
\hline 39. 8-Isopentenyl-kaempferol ${ }^{b}$ & 354.38 & 3.63 & 4 & 6 & 38.04 & 0.39 & $\mathrm{HE}$ \\
\hline $\begin{array}{l}\text { 40. Anhydroicaritin(3,5,7-trihydroxy-2-(4-methoxyphenyl)-8-(3-methylbut-2-enyl)chromen-4- } \\
\text { one) }\end{array}$ & 368.41 & 3.88 & 3 & 6 & 45.41 & 0.44 & $\mathrm{HE} / \mathrm{RA}$ \\
\hline 41. Suchilactone ${ }^{a}$ & 368.41 & 3.73 & 0 & 6 & 57.52 & 0.56 & RAS \\
\hline $\begin{array}{l}\text { 42. 6-Hydroxy-11,12-dimethoxy-2,2-dimethyl-1,8-dioxo-2,3,4,8-tetrahydro-43.1 H- } \\
\text { isochromeno[3,4-h]isoquinolin-2-ium }\end{array}$ & 370.41 & 2.75 & 1 & 6 & 60.64 & 0.66 & HE \\
\hline 43. Ohioensin- $A^{a}$ & 372.39 & 3.57 & 3 & 5 & 38.13 & 0.76 & $\mathrm{RMO}$ \\
\hline 44. Phellavin_qt ${ }^{\mathrm{a}}$ & 374.42 & 2.51 & 5 & 7 & 35.86 & 0.44 & $\mathrm{CPC}$ \\
\hline 45. Olivil $^{\mathrm{a}}$ & 376.44 & 1.68 & 4 & 7 & 62.23 & 0.41 & HE \\
\hline 46. Jatrorrhizine $e^{a, b}$ & 380.5 & 4.44 & 1 & 4 & 30.44 & 0.75 & CPC \\
\hline $\begin{array}{l}\text { 47. Stigmasterol((3S,8S,9S,10R,13R,14S,17R)-17-[(2R,5S)-5-ethyl-6-methylhept-3-en-2-yl]-10,13- } \\
\text { dimethyl-2,3,4,7,8,9,11,12,14,15,16,17-dodecahydro-1H-cyclopenta[a]phenanthren-3-ol) })^{b}\end{array}$ & 412.77 & 7.64 & 1 & 1 & 43.83 & 0.76 & $\mathrm{RC}$ \\
\hline 48. Diosgenin $((3 \beta, 25 R) \text {-spirost-5-en-3-ol })^{b}$ & 414.69 & 4.63 & 1 & 3 & 80.88 & 0.81 & RA \\
\hline $\begin{array}{l}\text { 49. ZINC03982454((3R,8S,9S, 10R,13R,14R,17R)-17-[(2R,5S)-5-ethyl-6-methylheptan-2-yl]-10,13- } \\
\text { dimethyl-2,3,4,7,8,9,11,12,14,15,16,17-dodecahydro-1H-cyclopenta[a]phenanthren-3-ol) }\end{array}$ & 414.79 & 8.08 & 1 & 1 & 36.91 & 0.76 & $\mathrm{RC}$ \\
\hline $\begin{array}{l}\text { 50. Beta-sitosterol(17-(5-Ethyl-6-methylheptan-2-yl)-10,13-dimethyl- } \\
\text { 2,3,4,7,8,9,11,12,14,15,16,17-dodecahydro-1 H-cyclopenta[a]phenanthren-3-ol) }{ }^{\mathrm{b}}\end{array}$ & 414.79 & 8.08 & 1 & 1 & 36.91 & 0.75 & $\mathrm{RC}$ \\
\hline 51. Timosaponin B III_qt ${ }^{a}$ & 416.71 & 4.77 & 2 & 3 & 35.26 & 0.87 & RA \\
\hline 52. Anemarsaponin C_qt ${ }^{a}$ & 416.71 & 4.97 & 2 & 3 & 35.50 & 0.87 & RA \\
\hline $\begin{array}{l}\text { 53. Phyllanthin(4-[(2S,3S)-3-[(3,4-dimethoxyphenyl)methyl]-4-methoxy-2-(methoxymethyl) } \\
\text { butyl]-1,2-dimethoxybenzene) }\end{array}$ & 418.58 & 4.11 & 0 & 6 & 33.31 & 0.42 & RAS \\
\hline 54. Yinyanghuo $A^{a}$ & 420.49 & 4.20 & 3 & 6 & 56.96 & 0.77 & HE \\
\hline 55. Cycloartenol (9beta,19-Cyclo-24-lanosten-3beta-ol) ${ }^{\text {b }}$ & 426.80 & 7.55 & 1 & 1 & 38.69 & 0.78 & $\mathrm{RC}$ \\
\hline 56. Anemarsaponin F_qt ${ }^{\mathrm{a}}$ & 432.71 & 3.92 & 2 & 4 & 60.06 & 0.79 & RA \\
\hline 57. Asperglaucide(aurantiamide acetate) & 444.57 & 4.02 & 2 & 6 & 58.02 & 0.52 & RA \\
\hline 58. Anemarsaponin E_qt ${ }^{a}$ & 448.76 & 4.53 & 2 & 4 & 30.67 & 0.86 & RA \\
\hline 59. Obacunone $e^{a, b}$ & 454.56 & 2.68 & 0 & 7 & 43.29 & 0.77 & CPC \\
\hline $\begin{array}{l}\text { 60. Icariside } A 7((2 R, 3 S, 4 S, 5 R, 6 S) \text {-2-(hydroxymethyl)-6-(7-hydroxy-3,4,6-trimethoxyphenan- } \\
\text { thren-2-yl)oxyoxane-3,4,5-triol0 }\end{array}$ & 462.49 & 1.16 & 5 & 10 & 31.91 & 0.86 & HE \\
\hline 61. Hispidone ${ }^{a}$ & 472.78 & 4.46 & 2 & 4 & 36.18 & 0.83 & CPC \\
\hline 62. Kihadanin $A^{a}$ & 486.56 & 1.76 & 1 & 9 & 31.60 & 0.70 & $\mathrm{CPC}$ \\
\hline 63. Isoprincepin ${ }^{a}$ & 494.53 & 2.52 & 5 & 9 & 49.12 & 0.77 & $\mathrm{RMO}$ \\
\hline
\end{tabular}

HE herba Epimedium Brevicornum, RC Rhizoma Curculiginis Orchioides, RMO Radix Morindae Officinalis, RAS Radix Angelicae Sinensis, CPC Cortex Phellodendri Chinensis, RA Rhizoma Anemarrhenae Asphodeloides.

a IUPAC name were not provided in database.

b 20 EXD bioactive compounds related to 34 significant pathway- or 12 gene- associated with menopause. 


\section{Revealing the significant pathways and gene-associated diseases}

Overall, 155 of the 721 compounds from EXD were found to have 2,656 chemical-protein interactions. After removing the overlapping/duplicate information, 1,963 associated proteins were obtained (Additional file 2). 1,824 of 1,963 proteins with a confidence score exceeding 0.5 were obtained. After enrichment analysis of 1,824 associated proteins, XD-scores and q values of pathways have been obtained. The XD-score is relative to the average distance to all pathways and represents a deviation from the average distance [43]. A larger positive XD-score indicates a stronger association between the inputted associated proteins and molecular interaction network of pathways. The $\mathrm{q}$ value determines the significance of the overlap (Fisher's exact test) between the input information and the pathways. The enrichment algorithm analysis (graphbased statistic) of XD-score and q-value revealed that the threshold value of XD-score in this study was 0.67 , therefore there are 34 pathways significantly associated with input set of proteins (Table 3).

In total, 210 of the 721 compounds from EXD were found to have 14,893 compound-gene interactions with 8,536 associated genes in the CTD (Additional file 3). Subsequently, the 8,536 genes were ranked according to their frequency of occurrence. The number of genes fell abruptly when the frequency of occurrence was small (gene frequency $\leq 8$; Figure 2). Subsequently, the number of genes became stabilized for gene frequencies between 10 and 19. However, the number of genes with gene frequencies $\geq 20$ was quite small. Genes with gene frequencies below the average of 1.74 were removed to reduce the number of redundant genes. After that, the remaining 2,183 genes were used to conduct the gene enrichment analysis by the DAVID platform. The "GENETIC_ASSOCIATION_DB_ DISEASE_CLASS" was selected as the annotation category to search for the significant diseases associated with the input genes, which was statistically verified by Fisher's exact test using the DAVID platform [47]. $P \leq 0.01$ indicated significant association or enrichment with the related items. After removing nonspecific diseases, 12 classes of diseases were found to be highly associated with the input genes (Tables 4 and 5). Most of these diseases were related to menopause, such as aging, reproduction, cancer, cardiovascular diseases, and neurological diseases [55-58].

\section{Identifying twenty bioactive compounds related} to menopause with following the druggability prediction

Eighteen of the 155 compounds that have 2,656 chemical-protein interaction, followed the Lipinski's Rule with $\mathrm{OB} \geq 30 \%$ and DL index $\geq 0.18$. Thirteen of the 210 compounds that have compound-gene interactions interaction, followed the Lipinski's Rule with $\mathrm{OB} \geq 30 \%$ and DL index
Table 3 The 34 significant pathways found by JEPETTO (Cytoscape plugin) with KEGG database

\begin{tabular}{|c|c|c|c|}
\hline Pathway & XD-score & q value & Overlap/size \\
\hline Linoleic acid metabolism & 3.148 & 0.000 & $10 / 11$ \\
\hline Citrate cycle (TCA cycle) & 3.057 & 0.000 & $21 / 26$ \\
\hline Propanoate metabolism & 1.899 & 0.000 & $11 / 19$ \\
\hline Arachidonic acid metabolism & 1.865 & 0.000 & $17 / 26$ \\
\hline PPAR signaling pathway & 1.794 & 0.000 & $22 / 39$ \\
\hline Tyrosine metabolism & 1.475 & 0.001 & $9 / 17$ \\
\hline Retinol metabolism & 1.474 & 0.009 & $6 / 12$ \\
\hline Bladder cancer & 1.463 & 0.000 & $17 / 38$ \\
\hline Ether lipid metabolism & 1.370 & 0.002 & $8 / 16$ \\
\hline $\begin{array}{l}\text { Metabolism of xenobiotics by } \\
\text { cytochrome P450 }\end{array}$ & 1.313 & 0.001 & $10 / 20$ \\
\hline Adipocytokine signaling pathway & 1.248 & 0.000 & $24 / 57$ \\
\hline $\begin{array}{l}\text { Drug metabolism: cytochrome } \\
\text { P450 }\end{array}$ & 1.240 & 0.003 & $8 / 17$ \\
\hline Fatty acid metabolism & 1.096 & 0.000 & $12 / 26$ \\
\hline Pyruvate metabolism & 1.096 & 0.000 & $12 / 26$ \\
\hline One carbon pool by folate & 1.057 & 0.018 & $5 / 10$ \\
\hline $\begin{array}{l}\text { Glyoxylate and dicarboxylate } \\
\text { metabolism }\end{array}$ & 1.057 & 0.018 & $5 / 10$ \\
\hline Fc epsilon RI signaling pathway & 1.040 & 0.000 & $23 / 65$ \\
\hline Pancreatic cancer & 1.013 & 0.000 & $25 / 70$ \\
\hline Steroid hormone biosynthesis & 0.990 & 0.027 & $6 / 15$ \\
\hline GnRH signaling pathway & 0.969 & 0.000 & $32 / 83$ \\
\hline Beta-Alanine metabolism & 0.924 & 0.007 & $7 / 15$ \\
\hline Prostate cancer & 0.913 & 0.000 & $32 / 84$ \\
\hline Tryptophan metabolism & 0.903 & 0.000 & $12 / 26$ \\
\hline Long-term depression & 0.893 & 0.000 & $23 / 57$ \\
\hline Toll-like receptor signaling pathway & 0.883 & 0.000 & $32 / 90$ \\
\hline $\begin{array}{l}\text { NOD-like receptor signaling } \\
\text { pathway }\end{array}$ & 0.879 & 0.000 & $21 / 59$ \\
\hline $\begin{array}{l}\text { Biosynthesis of unsaturated fatty } \\
\text { acids }\end{array}$ & 0.875 & 0.027 & $5 / 11$ \\
\hline Riboflavin metabolism & 0.875 & 0.027 & $5 / 11$ \\
\hline VEGF signaling pathway & 0.872 & 0.000 & $24 / 62$ \\
\hline Glycerophospholipid metabolism & 0.829 & 0.002 & $13 / 35$ \\
\hline Type II diabetes mellitus & 0.814 & 0.010 & $13 / 43$ \\
\hline Chagas disease & 0.804 & 0.000 & $36 / 99$ \\
\hline Selenoamino acid metabolism & 0.763 & 0.049 & $6 / 17$ \\
\hline Renal cell carcinoma & 0.708 & 0.000 & $24 / 68$ \\
\hline
\end{tabular}

$\geq 0.18$. Finally, 11 compounds has been identified related to both chemical-gene and chemical-protein interaction and followed the druglikeness prediction. Moreover, 20 compounds related to 34 significant pathway- or 12 gene- associated with menopause have been identified (Table 3).

\section{Discussion}

The actions of bioactive compounds in EXD were investigated by combining a drug prediction method with an enrichment analysis using information from bioinformatics 


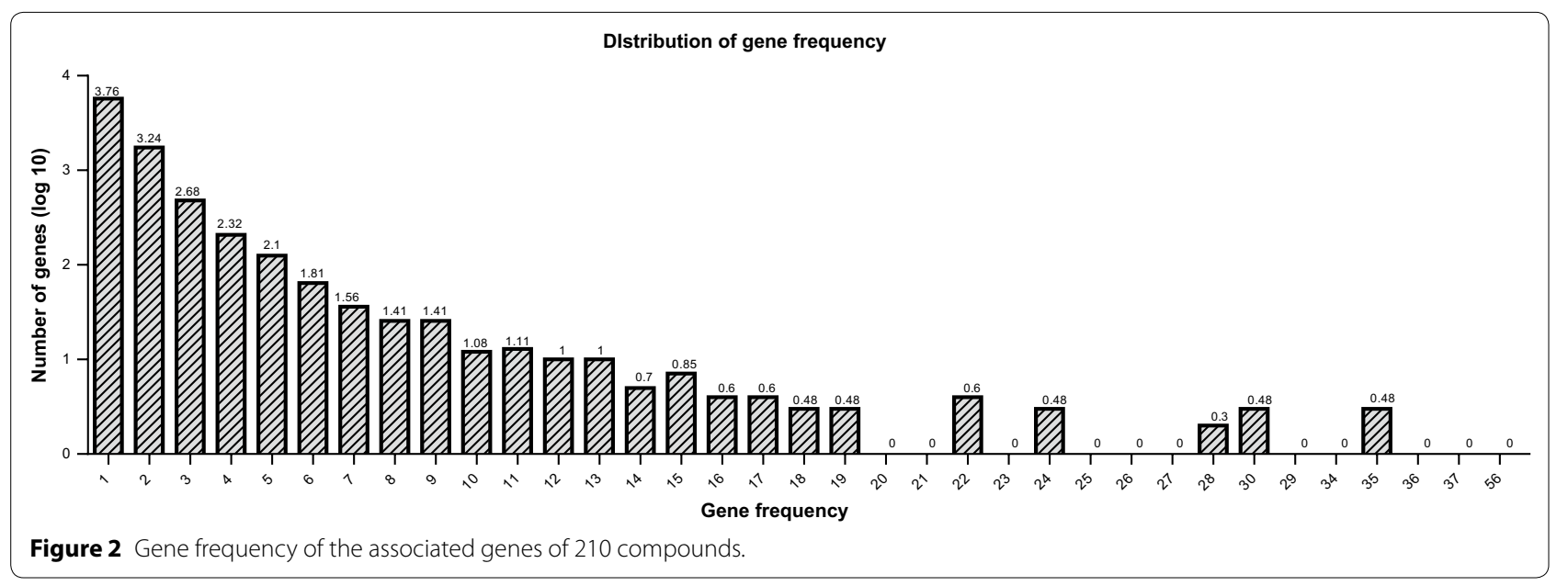

Table 4 Chemical-protein interactions and related significant signaling pathways

\begin{tabular}{|c|c|c|c|}
\hline Herb & Compound & Protein & Pathway \\
\hline $\mathrm{HE}$ & Emodin (1,3,8-trihydroxy-6-methylanthracene-9,10-dione) & HSD11B1 & Steroid hormone biosynthesis \\
\hline $\mathrm{RMO}$ & Alcool methylique & SULT2B1 & Steroid hormone biosynthesis \\
\hline CPC & Phenanthrene(TCMSP don't record IUPAC name) & CYP1A1 & Steroid hormone biosynthesis \\
\hline $\mathrm{RMO}$ & Caffeic acid ((Z)-3-(3,4-dihydroxyphenyl)prop-2-enoic acid) & COMT & Steroid hormone biosynthesis \\
\hline HE & Quercetin(2-(3,4-dihydroxyphenyl)-3,5,7-trihydroxychromen-4-one) & CYP19A1 & Steroid hormone biosynthesis \\
\hline $\mathrm{HE}$ & Quercetin-3-O-glucoside & CYP1B1 & Steroid hormone biosynthesis \\
\hline $\mathrm{HE}$ & Apigenin(5,7-dihydroxy-2-(4-hydroxyphenyl)chromen-4-one) & PTK2 & VEGF signaling pathway \\
\hline $\mathrm{RMO}$ & Hemo-sol((4R)-1-methyl-4-prop-1-en-2-ylcyclohexene) & HRAS & VEGF signaling pathway \\
\hline $\mathrm{HE} / \mathrm{RA}$ & Kaempferol(3,5,7-trihydroxy-2-(4-hydroxyphenyl)chromen-4-one) & MAPK1/AKT1/SRC & VEGF signaling pathway \\
\hline $\mathrm{RMO}$ & Esculetin(6,7-dihydroxychromen-2-one) & MAPK3 & VEGF signaling pathway \\
\hline $\mathrm{RMO}$ & Citric acid(2-hydroxypropane-1,2,3-tricarboxylic acid) & KRAS & VEGF signaling pathway \\
\hline $\mathrm{HE}$ & Oleanolic acid((3-beta)-3-Hydroxyolean-12-en-28-oic acid) & MAPK14 & VEGF signaling pathway \\
\hline RAS & Adenine(7H-purin-6-amine) & PTGS2 & VEGF signaling pathway \\
\hline $\mathrm{HE}$ & Emodin(1,8-dihydroxy-3-(hydroxymethyl)anthracene-9,10-dione) & VEGFA & VEGF signaling pathway \\
\hline $\mathrm{HE}$ & Luteolin(2-(3,4-dihydroxyphenyl)-5,7-dihydroxychromen-4-one) & PIK3CB & VEGF signaling pathway \\
\hline RA & 3,5,7-Trihydroxy-4'-methoxyl-8-prenylflavone-3-O-rhamnopyranoside & CASP9 & VEGF signaling pathway \\
\hline $\mathrm{RC} / \mathrm{RMO}$ & Myristic acid & РРP3СВ/РРРЗСА & VEGF signaling pathway \\
\hline $\mathrm{RC} / \mathrm{RMO} / \mathrm{RAS}$ & Palmitic acid & NOS3 & VEGF signaling pathway \\
\hline RAS & Lecithin & $\mathrm{PLA} 2 \mathrm{G} 4 \mathrm{~A}$ & VEGF signaling pathway \\
\hline $\mathrm{HE} / \mathrm{RMO}$ & Lauric acid & PIK3CA & VEGF signaling pathway \\
\hline RA & $\begin{array}{l}\text { Chinoinin(1,3,6,7-tetrahydroxy-2-[(2S,3R,4R,5S,6R)-3,4,5-trihydroxy-6-(hydroxymethyl) } \\
\text { oxan-2-yl]xanthen-9-one) }\end{array}$ & PLA2G2A & VEGF signaling pathway \\
\hline RAS & 4-Methoxybenzoic acid & PLA2G1B & VEGF signaling pathway \\
\hline $\mathrm{RMO}$ & Nonanoic acid & NFAT5/NFATC3 & VEGF signaling pathway \\
\hline $\mathrm{RMO}$ & $(9 Z, 12 Z)$-octadeca-9,12-dienoic acid & PLA2G10/PLA2G5 & VEGF signaling pathway \\
\hline $\mathrm{RC} / \mathrm{RMO}$ & Myristic acid & PPP3CC & VEGF signaling pathway \\
\hline
\end{tabular}

databases at the gene and protein levels. For example, candidate compounds such as berberine, palmatine, and jatrorrhizine, which we identified using our drug prediction method, have been shown to exhibit extensive pharmacological activities [59, 60]. From the enrichment analysis based on the available information for compound-protein and compound-gene interactions of EXD, we identified the most significantly related pathways and gene-associated disease, including pathways related to endocrine [35], VEGF [61], lipid metabolism [62] and anti-inflammatory [34]. Their pharmacological association with EXD were in line with previous publications $[34,35,61,62]$. 
Table 5 The 12 disease classes highly associated with input genes

\begin{tabular}{lccr}
\hline Disease class & Number of input genes involved in the disease & Input genes/total genes involved in the disease (\%) & $P$ value \\
\hline Cancer & 384 & 14.5 & $9.4 \mathrm{E}-28$ \\
Cardiovascular & 342 & 12.9 & $2.8 \mathrm{E}-25$ \\
Aging & 79 & 3.0 & $8.7 \mathrm{E}-17$ \\
Reproduction & 133 & 5.0 & $1.3 \mathrm{E}-11$ \\
Renal & 100 & 3.8 & $2.5 \mathrm{E}-10$ \\
Neurological & 247 & 9.3 & $1.9 \mathrm{E}-7$ \\
Infection & 142 & 5.4 & $2.0 \mathrm{E}-7$ \\
Psychological & 238 & 9.0 & $2.9 \mathrm{E}-7$ \\
Immune & 316 & 11.9 & $1.4 \mathrm{E}-5$ \\
Hematological & 70 & 2.6 & $1.4 \mathrm{E}-4$ \\
Vision & 85 & 3.2 & $4.4 \mathrm{E}-4$ \\
Developmental & 105 & 4.0 & $6.4 \mathrm{E}-3$ \\
\hline
\end{tabular}

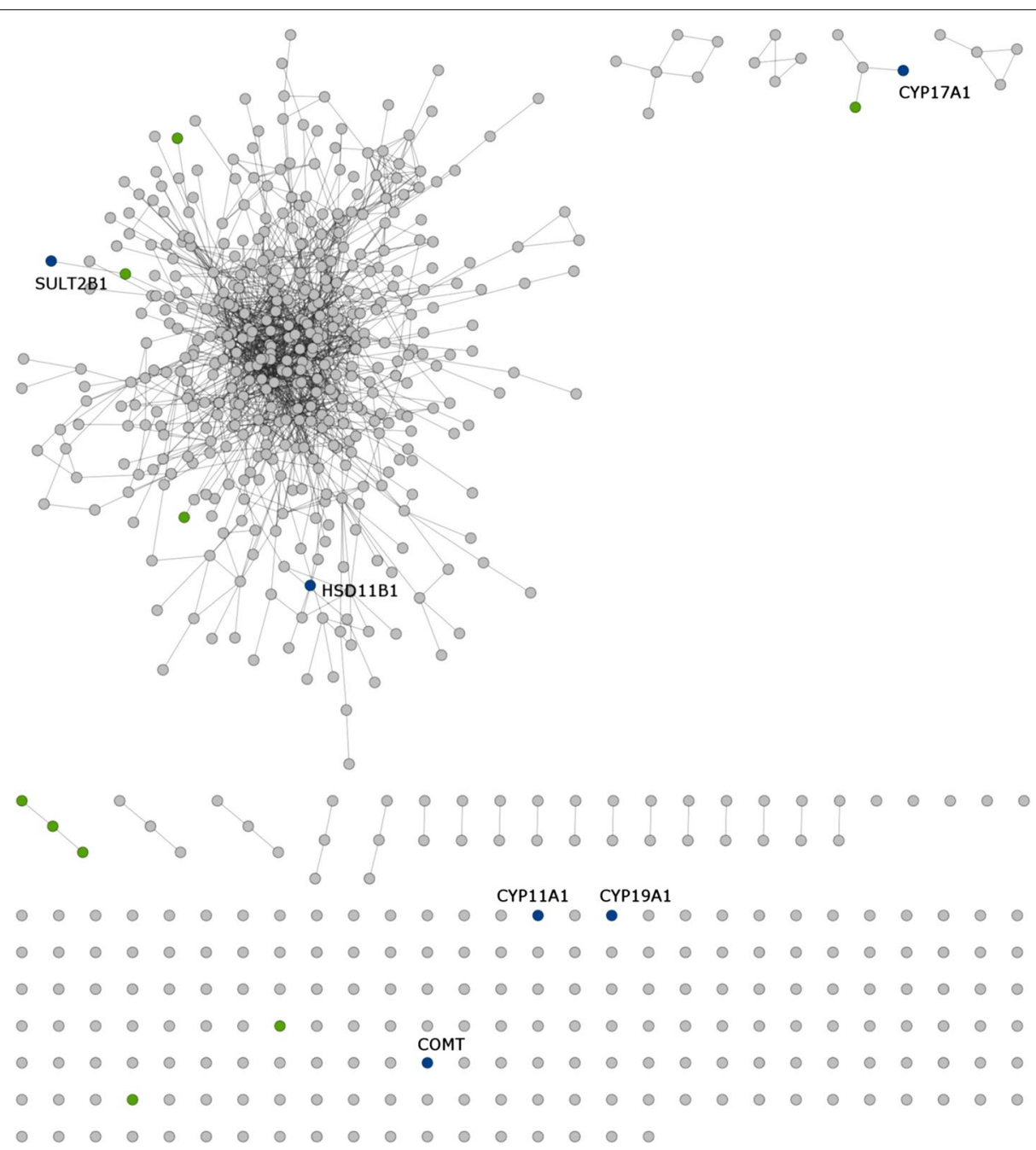

Figure 3 Chemical-protein interactions related to steroid hormone biosynthesis pathways. The grey color represents genes in the target set, green relates to the steroid hormone biosynthesis pathway, blue (labeled) is the overlap between the related pathway and the input protein set. 


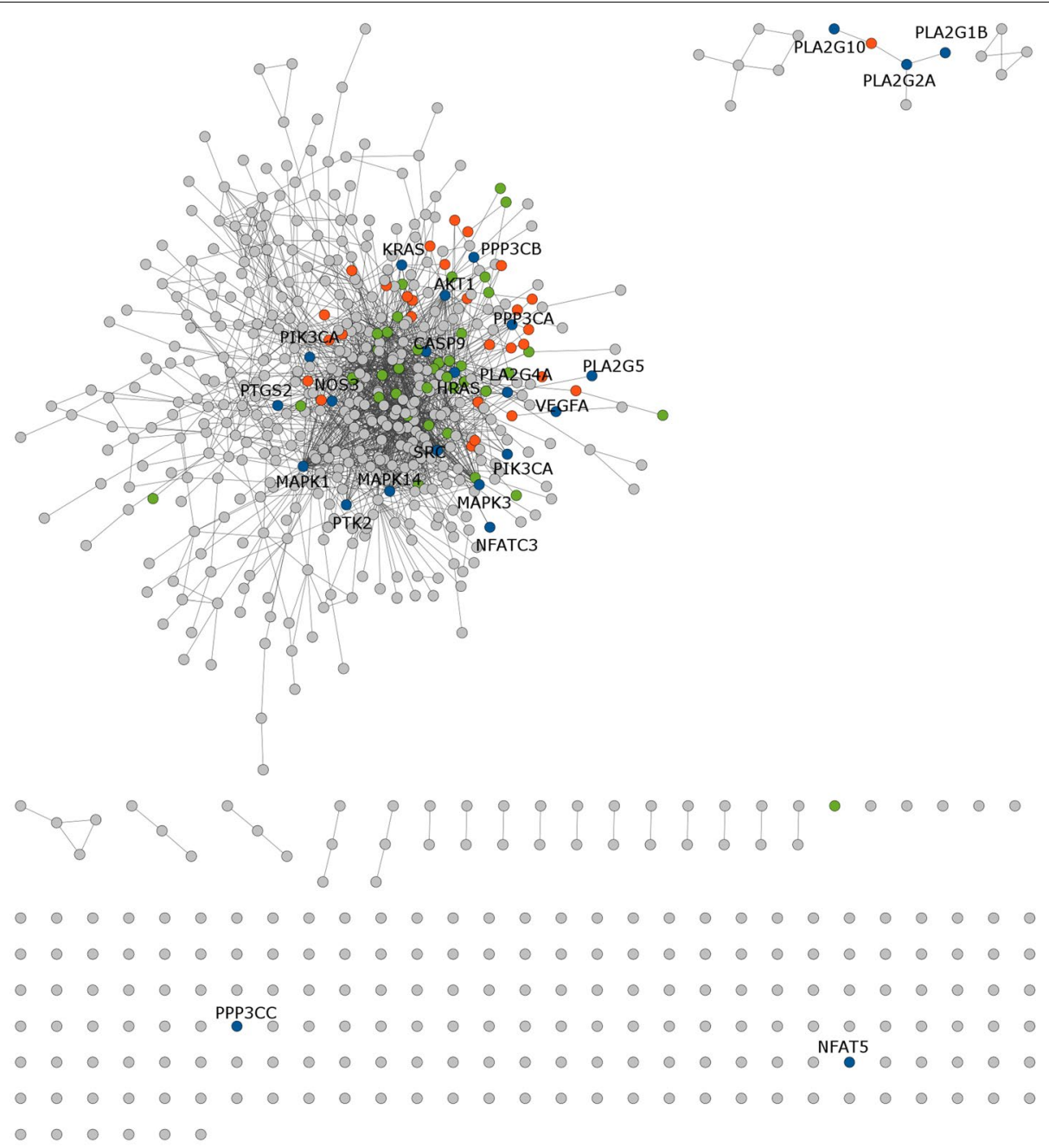

Figure 4 Chemical-protein interactions related to the VEGF signaling pathway. The grey color represents genes in the target set, green relates to the VEGF pathway, blue (labeled) is the overlap between the related pathway and the input protein set. The orange is the expansion of their pathways.

Several pathways involved the endocrine have also been identified, such as steroid hormone biosynthesis, GnRH signaling pathway, and adipocytokine signaling pathway, covering the previous finding of our group to promote estradiol biosynthesis in animal study [35]. For the steroid hormone biosynthesis signaling pathways, the EXD compound, quercetin, promoted the expression of aromatase (CYP19A1), which is the enzyme for estrogen biosynthesis [63]. This compound also met the druggability criteria. Other important overlapping proteins were HSD11B1, SULT2B1, CYP1A1, COMT, and CYP1B1 (Figure 3).

For the VEGF signaling pathways, VEGFA protein was involved in the antiangiogenic ability of EXD from our previous study [61]. The anti-cancer effect of EXD compound interact with VEGFA, emodin, has been reported [64]. Other interacting proteins of significance were
PTK2, HRAS, MAPK1, AKT1, SRC, MAPK3, KRAS, MAPK14, PTGS2, PIK3CB, CASP9, PPP3CB, PPP3CA, NOS3, PLA2G4A, PIK3CA, PLA2G2A, PLA2G1B, NFAT5, NFATC3, PLA2G10, PLA2G5, and PPP3CC (Figure 4). The steroid hormone biosynthesis and VEGF signaling pathways were selected for further analysis in the present study (Table 5).

For the lipid metabolism, EXD associated-pathways related to linoleic acid metabolism, fatty acid metabolism, unsaturated fatty acid biosynthesis, glycerophospholipid metabolism, arachidonic acid metabolism, and PPAR were identified [65-69]. Besides, our previous study found that EXD could improve the lipid profile in cardiovascular disease [62].

While a previous study showed EXD to have antiinflammatory activity [34], the present study suggested 
the pathways to include the Toll-like receptor signaling pathway, NOD-like receptor signaling pathway, and Fc epsilon RI signaling pathway [70-72]. This findings were consistent with previous studies on EXD antimetastatic activity in a human ovarian cancer model [73] and its antiangiogenic properties [61].

Compound-compound interactions were not considered in this study because the available databases could only provide limited information for the six individual herbs. The information of the databases did not cover the new compounds synthesized by chemical reactions during the decoction of EXD's ingredients, which will be confirmed by liquid chromatograph couple with mass spectrometry in further study. The ranking of the compound-gene and compound-protein interaction information was based on published evidence, but qualify of this evidence still needs extensive assessment. This study exemplified how to screen and identify bioactive compounds in CHFs.

\section{Conclusions}

Twenty compounds were identified by network pharmacology as potential effective ingredients of EXD for menopause with acceptable oral bioavailability and druggability.

\section{Additional files}

Additional file 1. Information on 721 identified compounds in EXD.

Additional file 2. Information on chemical-protein interaction pertaining to 155 compounds from EXD.

Additional file 3. Information on chemical-gene interaction pertaining to 210 compounds from EXD.

\footnotetext{
Abbreviations

EXD: Erxian decoction; CHFs: Chinese herbal formulas; TCMSP: traditional Chinese medicine systems pharmacology; STITCH: search tool for interactions of chemicals and proteins; CTD: The Comparative Toxicogenomics Database; LH: luteinizing hormone; FSH: follicle-stimulating hormone; HRT: hormone replacement therapy; CAM: complementary and alternative medicine; CM: Chinese medicine; OB: oral bioavailability; DL: drug-likeness; DAVID: The Database for Annotation, Visualization and Integrated Discovery; E2: estradiol; HSD11B1: hydroxysteroid (11-beta) dehydrogenase 1; SULT2B1: sulfotransferase family cytosolic 2B member 1; COMT: catechol-O-methyltransferase; CYP1A1: cytochrome P450, family 1, subfamily A, polypeptide 1; CYP1B1: cytochrome P450, family 1, subfamily B, polypeptide 1; CYP19A1: cytochrome P450, family 19, subfamily A, polypeptide 1;VEGF: vascular endothelial growth factor; GnRH: gonadotropin-releasing hormone; PTK2: protein tyrosine kinase 2; HRAS: Transforming protein p21; AKT1: RAC-alpha serine/threonine-protein kinase; MAPK1: mitogen-activated protein kinase 1; MAPK3: mitogen-activated protein kinase 3; SRC: SRC proto-oncogene, non-receptor tyrosine kinase; KRAS: GTPase KRas; CASP9: caspase-9; NOS3: nitric oxide synthase 3; PIK3CA: phosphatidylinositol-4,5-bisphosphate 3-kinase, catalytic subunit alpha isoform; PIK3CB: phosphatidylinositol-4,5-bisphosphate 3-kinase catalytic subunit beta isoform; PLA2G1B: phospholipase A2; PLA2G2A: membrane-associated phospholipase A2; PLA2G4A: cytosolic phospholipase A2; PLA2G5: calciumdependent phospholipase A2; PLA2G10: group 10 secretory phospholipase A2; NFAT5: nuclear factor of activated T-cells 5; NFATC3: nuclear factor of activated T-cells, cytoplasmic 3; PPP3CA: protein phosphatase 3, catalytic
}

subunit alpha isoform; PPP3CB: serine/threonine-protein phosphatase 2C catalytic subunit beta isoform; PPP3CC: serine/threonine-protein phosphatase 2B catalytic subunit gamma isoform.

\section{Authors' contributions}

SCWS, SWW, YBZ and TBN conceived and designed the study. SWW and SCWS searched the databases and conducted the network pharmacology study and analyses. SWW and SCWS wrote the manuscript. SCWS, JKWL, LXL and ZJZ revised the manuscript. All authors read and approved the final manuscript.

\section{Author details}

${ }^{1}$ School of Chinese Medicine, Li Ka Shing Faculty of Medicine, The University of Hong Kong, Hong Kong SAR, China. ${ }^{2}$ School of Biomedical Sciences, Faculty of Medicine, The Chinese University of Hong Kong, Hong Kong SAR, China. ${ }^{3}$ Department of Pharmacology and Pharmacy, Li Ka Shing Faculty of Medicine, The University of Hong Kong, Hong Kong SAR, China.

\section{Acknowledgements}

This study was partially supported by Grants from the Innovation and Technology Fund (project number ITS/262/09FP), the Innovation and Technology Commission, Government of Hong Kong Special Administrative Region; and Seed Funding Programme for Basic Research (project number 201211159146), the University of Hong Kong.

\section{Compliance with ethical guidelines}

Competing interests

The authors declare that they have no competing interests.

Received: 28 September 2014 Accepted: 1 July 2015

Published online: 19 July 2015

\section{References}

1. Cloutier CT, Coxworth JE, Hawkes K (2015) Age-related decline in ovarian follicle stocks differ between chimpanzees (Pan troglodytes) and humans. AGE 37:1-9

2. Burger HG, Dudley EC, Robertson DM, Dennerstein L (2002) Hormonal changes in the menopause transition. Recent Prog Horm Res 57:257-276

3. Landgren B-M, Collins A, Csemiczky G, Burger HG, Baksheev L, Robertson DM (2004) Menopause transition: annual changes in serum hormonal patterns over the menstrual cycle in women during a nine-year period prior to menopause. J Clin Endocrinol Metab 89:2763-2769

4. Dennerstein L, Dudley EC, Hopper JL, Guthrie JR, Burger HG (2000) A prospective population-based study of menopausal symptoms. Obstet Gynecol 96:351-358

5. Matthews KA, Wing RR, Kuller LH, Meilahn EN, Kelsey SF, Costello EJ et al (1990) Influences of natural menopause on psychological characteristics and symptoms of middle-aged healthy women. J Consult Clin Psychol 58:345

6. Avis NE, Stellato R, Crawford S, Johannes C, Longcope C (2000) Is there an association between menopause status and sexual functioning? Menopause 7:297-309

7. Society (2014) TNAM: terminology and statistics. http://www.menopause. org/docs/2012/cg_a.pdf?sfvrsn=2

8. Obermeyer CM, Reher D, Saliba M (2007) Symptoms, menopause status, and country differences: a comparative analysis from DAMES. Menopause 14:788-797

9. NIA: AgePage menopause. 2014. http://www.nia.nih.gov/health/ publication/menopause

10. NHS: menopause. 2014. http://www.nhs.uk/conditions/Menopause/ Pages/Introduction.aspx

11. Yang D, Haines C, Pan P, Zhang Q, Sun Y, Hong S et al (2008) Menopausal symptoms in mid-life women in southern China. Climacteric 11:329-336

12. Li L, Wu J, Pu D, Zhao Y, Wan C, Sun L et al (2012) Factors associated with the age of natural menopause and menopausal symptoms in Chinese women. Maturitas 73:354-360

13. Li Y, Yu Q, Ma L, Sun Z, Yang X (2008) Prevalence of depression and anxiety symptoms and their influence factors during menopausal transition and postmenopause in Beijing city. Maturitas 61:238-242 
14. Investigators WGftWsHI (2002) Risks and benefits of estrogen plus progestin in healthy postmenopausal women: principal results from the Women's Health Initiative randomized controlled trial. JAMA 288:321-333

15. Santen RJ, Allred DC, Ardoin SP, Archer DF, Boyd N, Braunstein GD et al (2010) Postmenopausal hormone therapy: an Endocrine Society scientific statement. J Clin Endocrinol Metab 95:1-66

16. Wang CC, Cheng KF, Lo WM, Law C, Li L, Leung PC et al (2013) A randomized, double-blind, multiple-dose escalation study of a Chinese herbal medicine preparation (Dang Gui Buxue Tang) for moderate to severe menopausal symptoms and quality of life in postmenopausal women. Menopause 20:241-249

17. Zhong LL, Tong Y, Tang GW, Zhang ZJ, Choi WK, Cheng KL et al (2013) A randomized, double-blind, controlled trial of a Chinese herbal formula (Er-Xian decoction) for menopausal symptoms in Hong Kong perimenopausal women. Menopause 20:767-776

18. Kang H, Ansbacher R, Hammoud M (2002) Use of alternative and complementary medicine in menopause. Int J Gynaecol Obstet 79:195-207

19. Hunt KJ, Coelho HF, Wider B, Perry R, Hung S, Terry R et al (2010) Complementary and alternative medicine use in England: results from a national survey. Int J Clin Pract 64:1496-1502

20. Posadzki P, Ernst E (2013) Prevalence of CAM use by UK climacteric women: a systematic review of surveys. Climacteric 16:3-7

21. Posadzki P, Lee MS, Moon TW, Choi TY, Park TY, Ernst E (2013) Prevalence of complementary and alternative medicine (CAM) use by menopausal women: a systematic review of surveys. Maturitas 75:34-43

22. Shen XH, Fang ZQ, Wu DX (1995) Effect of er-xian decoction and its disassembled prescription on enzyme activities and their gene expression of antioxidant enzymes in aging rat. Zhongguo Zhong Xi Yi Jie He Za Zhi 15:672-674

23. Dong BF, Fang ZQ, Shi JR (2006) Effect of er xian decoction and its subdivisions on granulosa cells secretory function in rats. Zhongguo Zhong Xi Yi Jie He Za Zhi 26(Suppl):122-125

24. Nian H, Qin LP, Zhang QY, Zheng HC, Yu Y, Huang BK (2006) Antiosteoporotic activity of Er-Xian Decoction, a traditional Chinese herbal formula, in ovariectomized rats. J Ethnopharmacol 108:96-102

25. Lu XN, Xu XR, Lin $\sqcup J$ (2008) Clinical observation of bushen er'xian decoction in treating premature ovarian failure. Zhongguo Zhong Xi Yi Jie He Za Zhi 28:594-596

26. Qin L, Han T, Zhang Q, Cao D, Nian H, Rahman Ket al (2008) Antiosteoporotic chemical constituents from Er-Xian Decoction, a traditional Chinese herbal formula. J Ethnopharmacol 118:271-279

27. Wu C, Zhang J, Zhou T, Guo B, Wang Y, Hou J (2011) Simultaneous determination of seven flavonoids in dog plasma by ultra-performance liquid chromatography-tandem mass spectrometry and its application to a bioequivalence study of bioactive components in Herba Epimedii and Er-Xian Decoction. J Pharm Biomed Anal 54:186-191

28. Xue L, Wang Y, Liu L, Zhao L, Han T, Zhang Q et al (2011) A HNMR-based metabonomics study of postmenopausal osteoporosis and intervention effects of Er-Xian Decoction in ovariectomized rats. Int J Mol Sci 12:7635-7651

29. Xue L, Jiao L, Wang Y, Nie Y, Han T, Jiang $Y$ et al (2012) Effects and interaction of icariin, curculigoside, and berberine in er-xian decoction, a traditional chinese medicinal formula, on osteoclastic bone resorption Evid Based Complement Alternat Med 2012:490843

30. Xue L, Wang Y, Jiang Y, Han T, Nie Y, Zhao L et al (2012) Comparative effects of er-xian decoction, epimedium herbs, and icariin with estrogen on bone and reproductive tissue in ovariectomized rats. Evid Based Complement Alternat Med 2012:241416

31. Wong KC, Lee KS, Luk HK, Wan HY, Ho CK, Zhang Y et al (2014) Er-xian Decoction exerts estrogen-like osteoprotective effects in vivo and in vitro. Am J Chin Med 42:409-426

32. Zhu Z, Xue LM, Han T, Jiao L, Qin LP, Li YS et al (2010) Antiosteoporotic effects and proteomic characterization of the target and mechanism of an Er-Xian Decoction on osteoblastic UMR-106 and osteoclasts induced from RAW264.7. Molecules 15:4695-4710

33. Yang Y, Chen MD, Li FY, Tang JF, Gao GF, Chen JL (2001) Effects of "two fairy decoction"(二仙湯) and its decomposed recipe on gonadotropinreleasing hormone secreted by GT1-7 cell line. Int Trad Chin West Med Pract Crit Care Med 8:143-145

34. Do W (2009) 40 Cases of clinical observation: the treatment of urinary tract infection by Erxian Decoction. Med Inform 22:1017-1018
35. Sze SC, Tong Y, Zhang YB, Zhang ZJ, Lau AS, Wong HK et al (2009) A novel mechanism: Erxian Decoction, a Chinese medicine formula, for relieving menopausal syndrome. J Ethnopharmacol 123:27-33

36. Hopkins AL (2008) Network pharmacology: the next paradigm in drug discovery. Nat Chem Biol 4:682-690

37. Hopkins AL (2007) Network pharmacology. Nat Biotech 25:1110-1111

38. Liang XJ, Li HY, Li S (2014) A novel network pharmacology approach to analyse traditional herbal formulae: the Liu-Wei-Di-Huang pill as a case study. Mol BioSyst 10:1014-1022

39. Azmi AS (2012) Network pharmacology for cancer drug discovery: are we there yet? Future Med Chem 4:939-941

40. Zhang GB, Li QY, Chen QL, Su SB (2013) Network pharmacology: a new approach for chinese herbal medicine research. Evid Based Complement Alternat Med 2013:621423

41. Zhao J, Jiang P, Zhang W (2010) Molecular networks for the study of TCM pharmacology. Brief Bioinform 11:417-430

42. Glaab E, Baudot A, Krasnogor N, Schneider R, Valencia A (2012) EnrichNet: network-based gene set enrichment analysis. Bioinformatics 28:i451-i457

43. Winterhalter C, Widera P, Krasnogor N (2014) JEPETTO: a Cytoscape plugin for gene set enrichment and topological analysis based on interaction networks. Bioinformatics 30:1029-1030

44. Huang DW, Sherman BT, Lempicki RA (2009) Bioinformatics enrichment tools: paths toward the comprehensive functional analysis of large gene lists. Nucleic Acids Res 37:1-13

45. Hu YM, Su GH, Sze SC, Ye W, Tong Y (2010) Quality assessment of Cortex Phellodendri by high-performance liquid chromatography coupled with electrospray ionization mass spectrometry. Biomed Chromatogr 24:438-453

46. Hu YM, Wang YT, Sze SC, Tsang KW, Wong HK, Liu Q et al (2010) Identification of the major chemical constituents and their metabolites in rat plasma and various organs after oral administration of effective Erxian Decoction (EXD) fraction by liquid chromatography-mass spectrometry. Biomed Chromatogr 24:479-489

47. Da Wei Huang BTS, Lempicki RA (2008) Systematic and integrative analysis of large gene lists using DAVID bioinformatics resources. Nat Protoc 4:44-57

48. Lipinski CA, Lombardo F, Dominy BW, Feeney PJ (2012) Experimental and computational approaches to estimate solubility and permeability in drug discovery and development settings. Adv Drug Deliv Rev 64:4-17

49. Ursu O, Rayan A, Goldblum A, Oprea TI (2011) Understanding druglikeness. Wiley Interdiscip Rev Comput Mol Sci 1:760-781

50. Xu X, Zhang W, Huang C, Li Y, Yu H, Wang Y et al (2012) A novel chemometric method for the prediction of human oral bioavailability. Int J Mol Sci 13:6964-6982

51. Tao W, Xu X, Wang X, Li B, Wang Y, Li Y et al (2013) Network pharmacology-based prediction of the active ingredients and potential targets of Chinese herbal Radix Curcumae formula for application to cardiovascular disease. J Ethnopharmacol 145:1-10

52. Wang $X, X u X$, Tao W, Li Y, Wang Y, Yang L (2012) A systems biology approach to uncovering pharmacological synergy in herbal medicines with applications to cardiovascular disease. Evid Based Complement Alternat Med 2012:1-15

53. Kuhn M, Szklarczyk D, Pletscher-Frankild S, Blicher TH, von Mering C, Jensen LJ et al (2014) STITCH 4: integration of protein-chemical interactions with user data. Nucleic Acids Res 42:401-407

54. Dai W, Chen J, Lu P, Gao Y, Chen L, Liu X et al (2013) Pathway patternbased prediction of active drug components and gene targets from H1N1 influenza's treatment with Maxingshigan-Yinqiaosan formula. Mol BioSyst 9:375-385

55. Wu JM, Zelinski MB, Ingram DK, Ottinger MA (2005) Ovarian aging and menopause: current theories, hypotheses, and research models. Exp Biol Med 230:818-828

56. Kelsey JL, Gammon MD, John EM (1993) Reproductive factors and breast cancer. Epidemiol Rev 15:36

57. Dosi R, Bhatt N, Shah P, Patell R (2014) Cardiovascular disease and menopause. J Clin Diagn Res 8:62

58. Shuster LT, Rhodes DJ, Gostout BS, Grossardt BR, Rocca WA (2010) Premature menopause or early menopause: long-term health consequences. Maturitas 65:161-166

59. Zhang AH, Sun H, Yang B, Wang XJ (2012) Predicting new molecular targets for rhein using network pharmacology (retracted article. See vol. $8,105,2014)$. BMC Syst Biol 6 
60. Bhadra K, Kumar GS (2011) Interaction of berberine, palmatine, coralyne, and sanguinarine to quadruplex DNA: a comparative spectroscopic and calorimetric study. Biochim Biophys Acta 1810:485-496

61. Yu X, Tong Y, Kwok HF, Sze SC, Zhong L, Lau CB et al (2012) Anti-angiogenic activity of Erxian Decoction, a traditional Chinese herbal formula, in zebrafish. Biol Pharm Bull 35:2119-2127

62. Sze SC, Cheung HP, Ng TB, Zhang ZJ, Wong KL, Wong HK et al (2011) Effects of Erxian decoction, a Chinese medicinal formulation, on serum lipid profile in a rat model of menopause. Chin Med 6:40

63. Xu L, Sun L, Lu L, Zhong X, Ma Y, Qin J (2014) Effects of quercetin on CYP450 and cytokines in aroclor 1254 injured endometrial cells of the pregnant rats. BioMed Res Int 2014:497-508

64. Hsu S-C, Chung J-G (2012) Anticancer potential of emodin. BioMedicine 2:108-116

65. West DB, Delany JP, Camet PM, Blohm F, Truett AA, Scimeca J (1998) Effects of conjugated linoleic acid on body fat and energy metabolism in the mouse. Am J Physiol 275:R667-R672

66. Bleijerveld OB, Houweling M, Thomas MJ, Cui Z (2006) Metabolipidomics: profiling metabolism of glycerophospholipid species by stable isotopic precursors and tandem mass spectrometry. Anal Biochem 352:1-14
67. Sampath $H$, Ntambi JM (2005) Polyunsaturated fatty acid regulation of genes of lipid metabolism. Annu Rev Nutr 25:317-340

68. Alves Martins D, Rocha F, Martínez-Rodríguez G, Bell G, Morais S, Castanheira F et al (2012) Teleost fish larvae adapt to dietary arachidonic acid supply through modulation of the expression of lipid metabolism and stress response genes. Br J Nutr 108:864-874

69. Wahli W, Michalik L (2012) PPARs at the crossroads of lipid signaling and inflammation. Trends Endocrinol Metab 23:351-363

70. Chen R, Alvero AB, Silasi DA, Mor G (2007) Inflammation, cancer and chemoresistance: taking advantage of the toll-like receptor signaling pathway. Am J Reprod Immunol 57:93-107

71. Fritz JH, Ferrero RL, Philpott DJ, Girardin SE (2006) Nod-like proteins in immunity, inflammation and disease. Nat Immunol 7:1250-1257

72. Field KA, Holowka D, Baird B (1995) Fc epsilon RI-mediated recruitment of p53/56lyn to detergent-resistant membrane domains accompanies cellular signaling. Proc Natl Acad Sci 92:9201-9205

73. Chu ES, Sze SC, Cheung HP, Liu Q, Ng TB, Tong Y (2013) An in vitro and in vivo investigation of the antimetastatic effects of a Chinese medicinal decoction, erxian decoction, on human ovarian cancer models. Integr Cancer Ther 12:336-346

\section{Submit your next manuscript to BioMed Central and take full advantage of:}

- Convenient online submission

- Thorough peer review

- No space constraints or color figure charges

- Immediate publication on acceptance

- Inclusion in PubMed, CAS, Scopus and Google Scholar

- Research which is freely available for redistribution

Submit your manuscript at

www.biomedcentral.com/submit

(O) Biomed Central 